\title{
Testing and characterization of challenging optics and optical systems with Shack Hartmann wavefront sensors
}

\author{
Julie Siv ${ }^{1}$, Rafael Mayer, Guillaume Beaugrand, Guillaume Tison, Rémy Juvénal, Guillaume Dovillaire \\ ${ }^{1}$ Imagine Optic, 18 rue Charles de Gaulle 91400 Orsay, France
}

\begin{abstract}
The Shack-Hartman wavefront sensor is a common metrology tool in the field of laser, adaptive optics and astronomy. However, this technique is still scarcely used in optics and optical system metrology. With the development of manufacturing techniques and the increasing need for optical characterization in the industry, the Shack-Hartmann wavefront sensor emerges as an efficient complementary tool to the wellestablished Fizeau interferometry for optical system metrology. Moreover, the raise of smart vehicles equipped with optical sensors and augmented reality, the optical characterization of glass and transparent flat materials becomes an issue that can be addressed with Shack-Hartmann sensors. Aberration measurements of challenging optics will be presented such as optical filters, thin flat optics, aspheric lenses and large optical assemblies.
\end{abstract}

\section{Comparison between a Shack- Hartmann and a Fizeau interferometer}

\subsection{Shack-Hartmann Principle}

In a Shack-Hartmann (SH) [1] wavefront sensor a microlens array is placed in front of a detector and each illuminated microlens is focusing on a dedicated part of the detector. The position of each focal spot is compared to a reference position, determined during calibration, gives information about the local slope of the wavefront which is used to reconstruct the aberration map of an optical system.

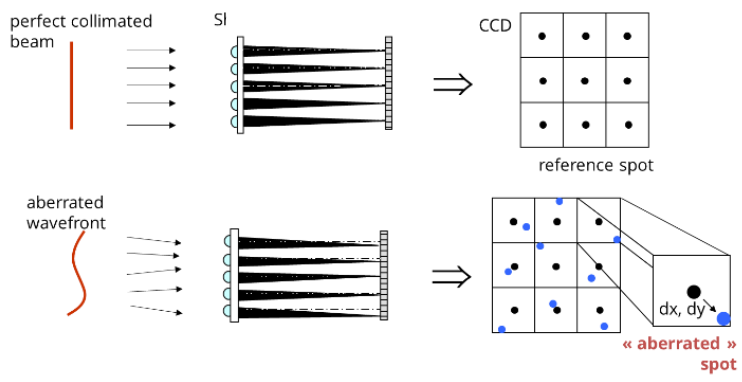

Fig. 1. Principle of Shack-Hartmann wavefront sensing, showing a perfect (top) and aberrated (bottom) wavefront.

\subsection{Comparison of a Shack-Hartmann wavefront sensor and a Fizeau interferometer}

We performed a comparison between a Fizeau interferometer and a SH wavefront sensor by characterizing a large flat substrate under the same conditions. To be able to do so, we used HASO RFLEX Large aperture, a metrology tool based on SH wavefront detection with an integrated light source and a collimator, allowing to make a measurement on a pupil of $100 \mathrm{~mm}$ in diameter.

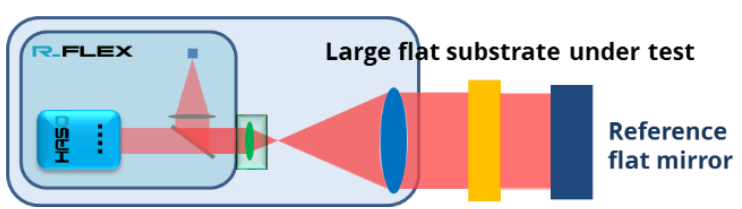

Fig. 2. Setup of the optical characterization of a large flat glass (yellow slab) with a Shack Hartmann wavefront sensor

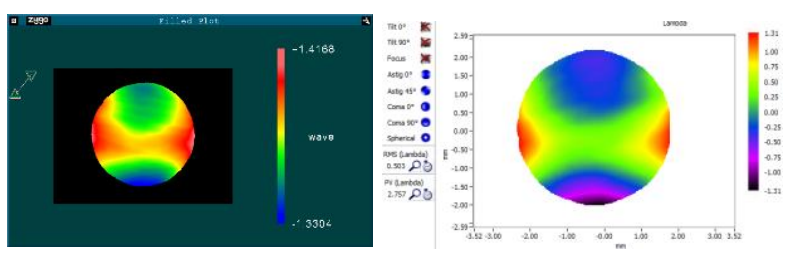

Fig. 3. (Left) measurement made with a Fizeau interferometer (reference model), the computed wavefront error is $2.74 \lambda \mathrm{PV}$. (Right) measurement made with a SH wavefront sensor, the computed wavefront error is $2.75 \lambda \mathrm{PV}$.

This result shows that there is an excellent agreement between the two measurements.

\section{Optics and optical system characterization and testing with $\mathrm{SH}$ wavefront sensors}

In this part we will show examples of aberration map of a set of optical components and systems that typically are difficult to measure with common interferometric measurements. These measurements were made with wavefront sensors from Imagine Optic and shows that SH wavefront sensors are good complementary tools for optics metrology.

\footnotetext{
* Corresponding author: jsiv@imagine-optic.com
} 


\subsection{Optical filters}

The SH wavefront sensor is achromatic. Thus, it is possible to characterize aberrations of an optical component according to the wavelength without changing the metrology tool.

We measured aberrations induced by a large optical filter in transmission at $632 \mathrm{~nm}$ and in reflection at $1064 \mathrm{~nm}$. We managed to measure the wedge and the curvature of the optics, which were the dominant aberrations. By filtering these two parameters, we have been able to measure the residual aberrations.
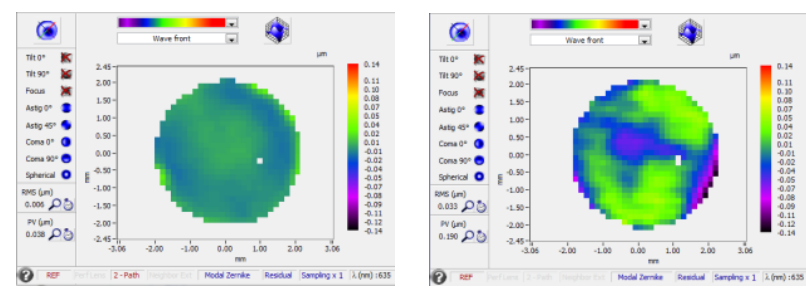

Fig. 4. (Left) Residual aberration in transmission at $635 \mathrm{~nm}$ $\mathrm{WFE}=6 \mathrm{~nm}$ RMS and (right) in reflection at $1064 \mathrm{~nm} \mathrm{WFE}=$ $32 \mathrm{~nm}$ RMS

\subsection{Glass and flat optics}

The SH technology is not based on interferometry. Thus, when measuring flat optics or glass, even for very thin samples, there is no parasite interferences due to Fabry Perot effect that would disturb the measurement. In the measurement bellow, it is possible to notice the effect of a black printing on the baking of the glass.
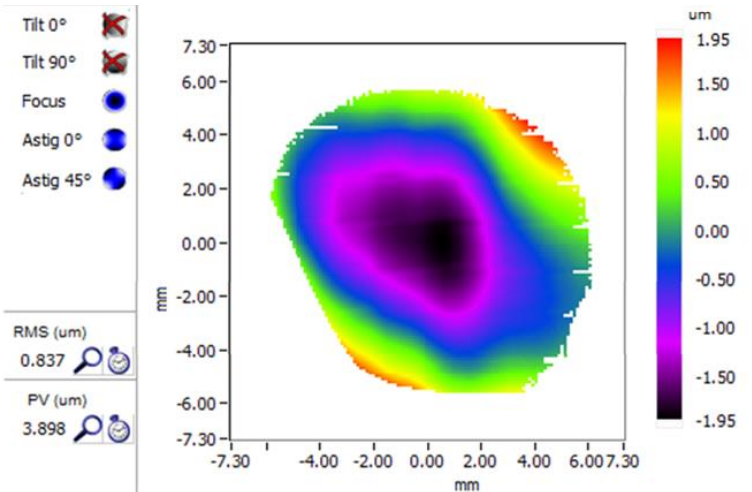

Fig. 5. SH wavefront map of a $2 \mathrm{~mm}$ thick glass with a block print edge. $\mathrm{WFE}=837 \mathrm{~nm}$ RMS

\subsection{Optical assemblies}

In some specific situations, characterization of an optical assembly requires a highly accurate measurement and a very compact sensor. In the case of large telescopes, the aberration is strongly dependant on its orientation because of the mechanical stress. That is why it is important to be able to design the metrology bench taking into account the orientation of the optical system.

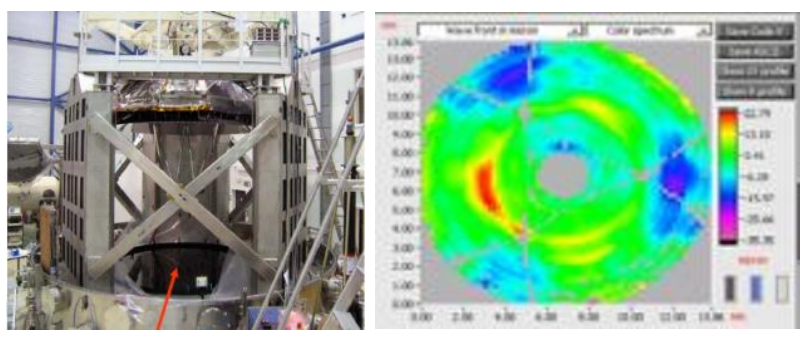

Fig. 6. Vertical optical characterization of Herschel telescope.

For the characterization of Herschel telescope, the HASO SH wavefront sensor was located below the telescope, and in this configuration, the wavefront map shows a non-neglectable trefoil aberration due to mechanical stress of the mirrors.

\subsection{Aspheric lenses and freeform optics}

With the advance of manufacturing techniques, freeform optics and aspheric lenses become more common. Thanks to its high dynamics - up to $3400 \lambda \mathrm{PV}$ for HASO4 120 - and its absolute measurement capability, the SH wavefront sensor is an ideal tool for characterization of free from and aspherical optics.

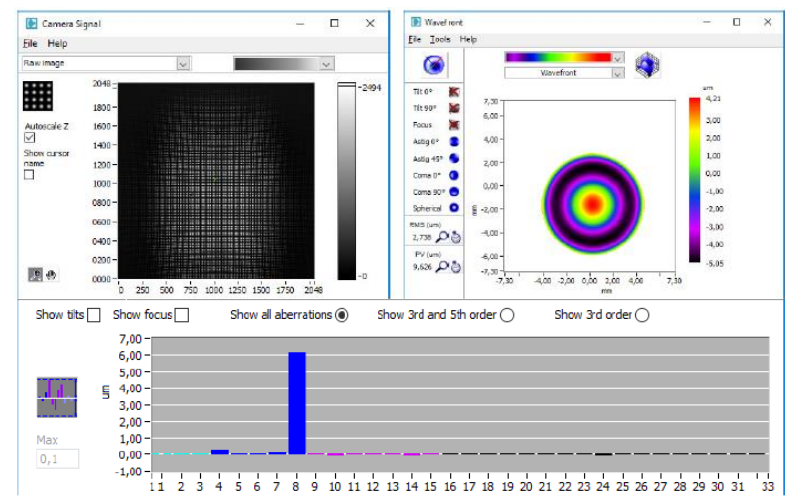

Fig. 7. Camera signal, wavefront and modal decomposition of a miniature aspheric lens.

\section{Conclusion}

Thanks to its features, the Shack Hartmann wavefront sensor is a good alternative for optics characterization in specific situations when the Fizeau interferometer fails at providing satisfying results.

Table 1 - Comparison between Shack Hartmann wavefront sensor and Fizeau interferometer.

\begin{tabular}{|c|c|}
\hline SH sensor & Fizeau interferometer \\
\hline Compact and portable & $\begin{array}{c}\text { Table top on an isolated } \\
\text { platform }\end{array}$ \\
\hline Dynamic of tens of $\mu \mathrm{m}$ & Dynamic of a few $\mu \mathrm{m}$ \\
\hline Limited spatial resolution & High spatial resolution \\
\hline Multi-wavelength capability & Single wavelength \\
\hline No reference beam & With a reference beam \\
\hline
\end{tabular}

\section{References}

1. Platt, Ben C.; Shack, R, Journal of Refractive Surgery. 17 (5): S573-7. PMID 11583233 (2001) 